\title{
Energy efficiency in double disc refining -Influence of intensity by segment design
}

Dino Muhić, Juha-Pekka Huhtanen, Lars Sundström, Christer Sandberg, Mats Ullmar, Petteri Vuorio, and Per Engstrand

KEYWORDS: TMP, Double disc refining, Intensity, Segment design, Energy efficiency, Segment performance simulation

SUMMARY: The goal with this work was to study the effect of segment design on electrical energy consumption and pulp quality in double disc TMP production. Mill scale trials were performed with refiner segments from Metso, which were designed based on the fluid dynamics theory. The calculated intensity for the different segments was related to the measured pulp quality. Refining with the high intensity segments (Turbine $^{\mathrm{TM}}$ ) produced pulp with similar tensile index and a significantly higher specific light scattering coefficient at certain specific energy consumption when compared with the reference segments. One drawback with the high intensity segments was the limited operating window due to fibre cutting. The trials showed that segment performance can be modelled, which facilitates the development of new segment designs.

\begin{tabular}{l}
\hline ADDRESSES OF THE AUTHORS: Dino Muhić \\
(dino.muhic@metso.com) Metso Paper - Refiner \\
Segments, Vretenvägen 6, SE-17154 Solna, Sweden \\
Juha-Pekka Huhtanen (juha-pekka.huhtanen@tut.fi) \\
Tampere University of Technology, Energy and Process \\
Engineering Institute, Tampere, Finland \\
Lars Sundström (lars.sundstrom@holmenpaper.com) \\
Christer Sandberg (christer.sandberg@holmenpaper.com) \\
Holmen Paper AB Braviken Paper Mill, SE-60188 \\
Norrköping, Sweden, Mats Ullmar \\
(mats.ullmar@metso.com) Metso Paper, SE-85194 \\
Sundsvall, Sweden, Petteri Vuorio \\
(petteri.vuorio@metso.com) Metso Paper Oy, P.O. Box \\
125, FI-37601, Valkeakoski, Finland, Per Engstrand \\
(per.engstrand@miun.se) Mid Sweden University, FSCN, \\
Holmgatan 10, SE-85170 Sundsvall, Sweden \\
Corresponding author: Dino Muhić
\end{tabular}

All refiners are hydraulic flow machines in which complex flow conditions and heat transfer are combined with delicate wood fibre processing. Therefore, it is important to know the flow conditions inside the refiner in order to understand the process and to produce the required pulp quality. Moreover, it is important to connect refining conditions to achieved pulp properties. To do this, a new way to define refining intensity, by analyzing the flow conditions and thermo dynamical state inside the refiner (Huhtanen et al. 2009) is used in this study. The physical changes of fibre structure and flow conditions inside the refiner can be controlled by changing the segment design. Studies were made on how certain pulp qualities can be produced with higher energy efficiency by segment design.

\section{Fluid dynamical model}

The flow situation inside the refiner is divided into two parts: main flow in the radial direction along refiner grooves, and secondary flow inside the refiner grooves and between bars in the tangential direction. The main flow characteristics determine gross features and functionality of the refiner, while the secondary flow characteristics principally determine the refiner's pulp quality parameters. The above approach can be applied to any refiner, especially to high-consistency chip refiners (Huhtanen et al. 2009; Huhtanen 2004).

Earlier results (Huhtanen 2004) showed that segment pattern affects the refining efficiency: bars with a large angle of incident (pumping) produced relatively flat radial steam pressure profile and the re-circulation (backflow) decreased compared to non-pumping segments.

This gives a more even intensity distribution along the segment radius. A more even intensity distribution is a result of the decrease in heat and steam production which creates a weaker adverse pressure gradient between refining segments (less backflow), where heat and steam production decrease makes it easier to achieve similar fibre residence time. Thereby, the fibres undergo similar treatment.

In order to understand the refining process and design refiner segments that produce adequate pulp quality, it is important to understand the flow phenomena inside a refiner. It is also important to be able to calculate or compute some basic values of flow field (fluid velocities, mass and volume fractions, residence time, etc.) and thermodynamical state (pressure, temperature, etc.) of the flow medium. Moreover, it is important to be able to connect these values to refiner operating parameters (disc gap, production, power, axial load, dilution water, steam production, consistency, etc.) and to have a measure to describe how these values and parameters affect the produced pulp quality. For this reason, a refining intensity formula has been developed. This formula can be used in the evaluation of the efficiency of various types of segments at different refining conditions. (Huhtanen 2004; Huhtanen et al. 2009). 


\section{Intensity}

Several attempts have been made to model and explain what happens in the refining zone (Miles, May 1990; Härkönen et al. 2000; Sabourin et al. 1997, Atack et al. 1984). Refining intensity is one way of describing the character of the treatment the fibres are subjected to, i.e. the energy transfer per unit mass of fibres per impact. In this study, the fluid dynamical theory is used to calculate the minimum fibre residence time, which is one of the corner stones for the refining intensity definition (Eq l) by Hahtokari and Huhtanen (Huhtanen 2004; Huhtanen et al. 2004; Huhtanen et al. 2009).

$\mathrm{I}_{\mathrm{H \& H}}=(60 \times \mathrm{P}) /\left(\mathrm{n} \times \mathrm{N}_{\mathrm{b}} \times \mathrm{t}_{\mathrm{r}}\right)$

The intensity is the power consumption for every bar crossing given that all fibres are moving through the refiner with a certain calculated minimum fibre residence time; where intensity is $\left(I_{H \& H}\right)$, power consumption $(P)$, rotational speed in $\operatorname{rpm}(n)$, number of bars $\left(N_{b}\right)$ and the minimum fibre residence time $\left(t_{r}\right)$. The minimum fibre residence time is used to describe the flow phenomena inside the refiner. This theory could be a good tool for elucidating the refining segment role in chip refining because it considers the influence of segment design on steam and material flow.

Forces acting on the fibres in refining are not always easy to describe. The reason for this is difficulties in determining the position of where the most intense refining occurs and where the most energy is applied due to shifting in e.g. temperature, pulp and steam flow (Härkönen et al. 2003).

To simplify the description of forces applied on the fibre flock when it is passing a bar crossing (two bars passing each other and change the flock shape (Stationwala et al. 1992), the force can be divided into two major forces: normal and shear forces. Normal forces contribute mostly to internal fibrillation of the fibre wall. The shear force is a product of friction and corner force (arise over the leading edge of a refiner bar during an impact). Principally, shear forces cause external fibrillation. (Sanger, Ouellet 2002)

The minimum fibre residence time is calculated by applying fluid mechanics, where the position, speed and volume of material are taken into account. The calculations include also the effect of compression and shear forces.

\section{Refiner segments}

To refine fibres in a TMP process, patterned discs (refiner segments) are used as an energy transferring tool. This means that the segment design is of great importance.

Proper pattern design can lead to a reduction of specific energy consumption (Albert 2001; Vuorio,
Bergqvist 2003). Low energy segments (LE'M) and Turbine ${ }^{\mathrm{TM}}$ segments are two types of segments used for reduction of energy consumption to reach a certain CSF and to reduce the content of long fibres and shives in the pulp. These segments with pumping bar angles shorten the fibre residence time in the refiner gap. (Vuorio, Bergqvist 2003, Pynnönen, Samboon 2008) With the introduction of LE segments some problems may occur. For example, the reduction of long fibre content can cause problems such as low tear strength. However, fibre length can be better preserved by increasing thermal softening via increasing the refining pressure/temperature (Kure et al. 1999; Vuorio, Bergqvist 2003).

An example of energy saving in TMP production is the use of Turbine segments in single and conical disc refiners. The measured and simulated specific energy consumption was $20 \%$ lower compared to standard segments for both types of refiners, without negatively affecting the produced pulp quality. The reason for this saving is higher intensity due to more pumping segments. (Huhtanen et al. 2004)

$E q 1$ describes how the refining intensity can be altered by changing the rotational speed, number of segment bars, pulp consistency (affects $t_{r}$ ) and power input. Pulp and steam flow and number of bar crossings, which determines the pulp residence time, can be changed by adjusting the segment design (Kure et al. 1999; Vikman et al. 2003).

The radial flows of fibre and steam are important factors in controlling the refining process. Beside for factors defined in $E q l\left(P, n\right.$ and $\left.N_{b}\right)$, the fibre and steam flows are determined by the amount and height of dams and the open cross-sectional area. Dams in the grooves force the pulp to move towards the impact zone located at the bars.

Another important factor is the power consumption along the segment. How this power consumption is distributed in a DD refiner is not yet totally clarified; however, an attempt to model this power consumption is made in this article.

\section{Materials and Methods}

\section{Segment performance simulations}

Segment performance simulations were made in order to design segments and predict pulp properties. The calculation and simulation method has been presented by Huhtanen (2004) and Huhtanen et al. (2004). Some of the aspects that were taken into account were steam and fibre velocity, fibre residence time, power distribution along the segment radius, temperature between the segments etc. This procedure has a basis in the fluid dynamics, as referred in the introduction part. One of the goals was to get an even power distribution 
along the segments. The other one was to have a short fibre residence time to gain high intensity, while having high temperature in order to soften the fibre. Some of the segment simulations, performed in FORTRAN code are shown as results.

Please note that LE $^{\mathrm{TM}}$ and Turbine ${ }^{\mathrm{TM}}$ are trademarks for many different segment types, which means that results of one segment type to another can differ. The results presented in this study are for a given segment type (article number), see Appendix.

\section{Mill trials for evaluation of new segment design}

Full scale trials were made at one of the TMP lines at the Holmen Paper Braviken mill with Norway spruce as raw material. In this work, only the primary refining system of RGP68DD refiners (Metso) was investigated. The primary goal was to study the effect of new segment designs on electrical energy consumption and pulp quality. A comparison of electrical energy consumption between the different segment designs was made at similar tensile index, shown in Table 1. The test segments LE (DN72N849-850) and Turbine (DN72N861-862) were run for one (1) and five (5) days respectively at process conditions shown in Table 2a-c. The reference segment was Metso DN72N816-817. All tested segments had the same c-segments (inner) (DO52B036-037). The plate gap temperature is measured at radius $810 \mathrm{~mm}$.

As shown in Tables 1-2, some process variation did occur and the upper limit for energy input was different for the different segment types.

\section{Pulp and handsheet analysis}

Pulp properties were analyzed as following, Table 3. Samples were collected from the blow line after the refiner. A Rapid Köthen sheet former was used for making handsheets.

\section{Results and Discussion}

Comparison between reference-, LE- and Turbine segments is made regarding segment simulations. However, LE pulp quality results are not presented due to fibre cutting at similar SEC. The trial with the LE segment is a development step in this study.

\section{Segment performance simulations}

The LE and Turbine segments used in this study are more feeding and provide higher refining intensity comparing to the reference segment. The feeding action of LE and Turbine segments can be seen in the temperature profile simulations, Fig 1. The calculated residence time values are shown in Fig 2 .
Table 1. Three tested refining segments and their specific refining energy operating window.

\begin{tabular}{lll}
\hline Segment type & $\begin{array}{l}\text { SEC (min) } \\
\text { (kWh/adt) }\end{array}$ & $\begin{array}{l}\text { SEC (max) } \\
\text { (kWh/adt) }\end{array}$ \\
\hline Standard (ref) & 1200 & 2000 \\
LE segment & 1200 & 1700 \\
Turbine segment & 1240 & 1650 \\
\hline
\end{tabular}

Table 2a. Process data, reference segment.

\begin{tabular}{lr}
\hline Process parameters & Trial reference \\
\hline Segment operating time $(\mathrm{h})$ & $\sim 170$ \\
Production (adt/h) & $10.8 \pm 0.2$ \\
Consistency $(\%)$ & $35.6 \pm 3.4$ \\
Inlet and housing pressure (bar $(\mathrm{g}))$ & 4.4 \\
Motor load (MW) & $14.3-21.6$ \\
Temperature plate gap $\left({ }^{\circ} \mathrm{C}\right)$ & $166-171$ \\
\hline
\end{tabular}

Table 2b. Process data, LE segment.

\begin{tabular}{lr}
\hline Process parameters & \multicolumn{1}{l}{ Trial LE } \\
\hline Segment operating time (h) & $<20$ \\
Production (adt/h) & $11.9 \pm 0.3$ \\
Consistency (\%) & $40.8 \pm 0.5$ \\
Inlet and housing pressure (bar(g)) & 5.4 \\
Motor load (MW) & $17.7 \pm 0.4$ \\
Temperature plate gap $\left({ }^{\circ} \mathrm{C}\right)$ & 173 \\
\hline
\end{tabular}

Table 2c. Process data, Turbine segment

\begin{tabular}{lr}
\hline Process parameters & \multicolumn{1}{c}{ Trial Turbine } \\
\hline Segment operating time $(\mathrm{h})$ & $<140$ \\
Production (adt/h) & $10.4 \pm 0.3$ \\
Consistency (\%) & $39.3 \pm 1.4$ \\
Inlet and housing pressure (bar(g)) & 4.6 \\
Motor load (MW) & $14.5-18.3$ \\
Temperature plate gap $\left({ }^{\circ} \mathrm{C}\right)$ & $162-167$ \\
\hline
\end{tabular}

Table 3. ISO and SCAN pulp and handsheet measurement

\begin{tabular}{ll}
\hline Measurement & Method \\
\hline Pulp consistency & ISO 4119:1995 \\
& \\
Hot disintegration & SS-EN 2471 \\
Sheet basis weight & SS-EN ISO 536 \\
CSF & ISO 5267-2 \\
Rapid Köthen & ISO 5269-2 \\
Spec light scattering coeff & SS-ISO 9416 \\
Tensile index & SS-EN ISO 5270 \\
Paper conditioning ratio & SS-EN 20187- \\
Long fibre distribution and shive & Eurocon PulpEye, \\
content & optical fibre analyser \\
\end{tabular}




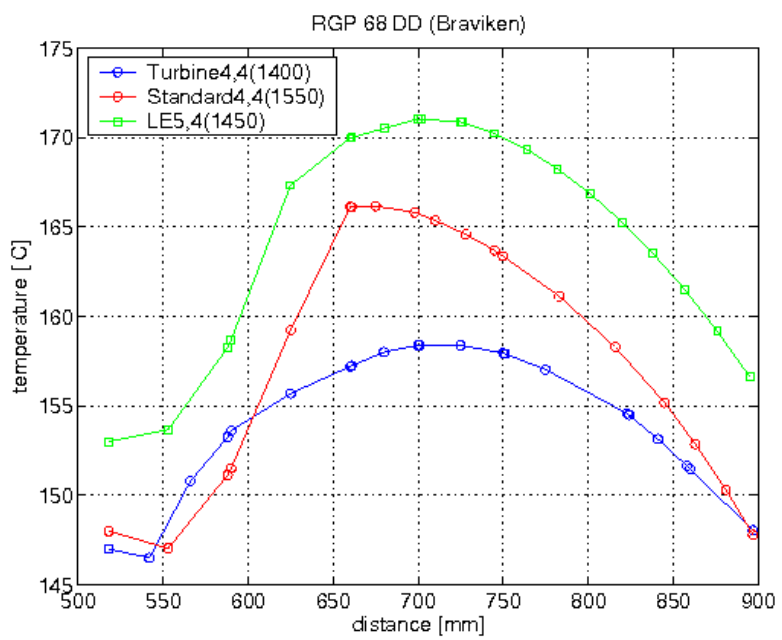

Fig 1. Temperature profile along the segment radius is shown for reference, LE and Turbine. Different SEC (within the brackets in the legend) is used in order to get comparable pulp quality results, e.g. fibre length and CSF. The Turbine segment has lower temperature and is less parabolic compared to other tested segments.

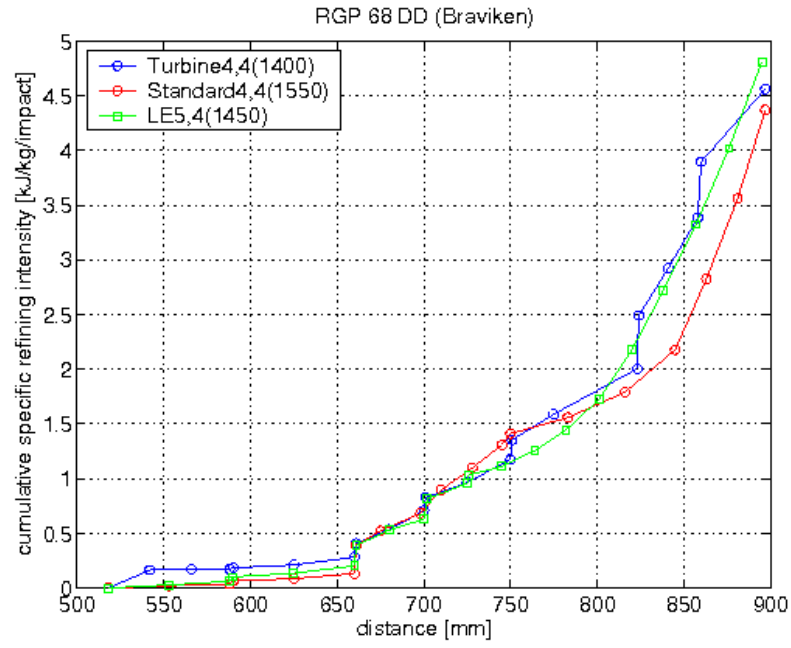

Fig 3. The cumulative refining intensity shows that Turbine and LE segments are treating the fibres at slightly higher intensity than the reference segments, even though the SEC is lower for both of them. However, it is the position (along the radius) of where the high intensity is applied that decides the pulp quality.

As Turbine segments are feeding pulp through the refiner faster than other tested segments, the residence time is shorter. Therefore, the Turbine refining intensity is higher than for the reference segment, even though Turbine segment specific energy consumption is lower, Fig 3. The intensity is distributed differently for the three tested type of segments. Turbine segments have higher intensity in the beginning of the segment while LE segments have a steeper intensity increase at the end. The overall intensity value for Turbine and LE is around $4.6 \mathrm{~kJ} / \mathrm{kg} / \mathrm{impact}$ compared to $4.4 \mathrm{~kJ} / \mathrm{kg} / \mathrm{impact}$ for the reference segment.

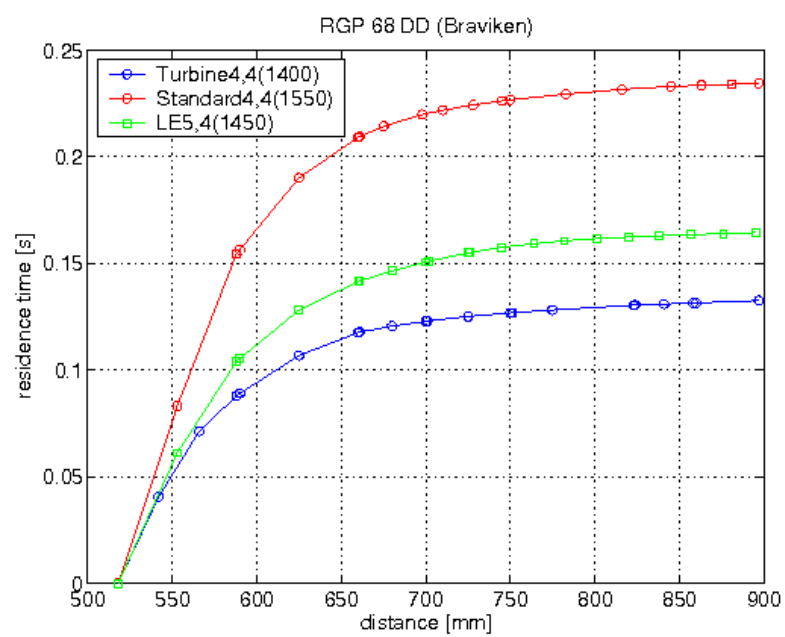

Fig 2. Illustration of calculated minimum fibre residence time. The Turbine segment minimum residence time is half of the reference, which shows that Turbine segments are more feeding.

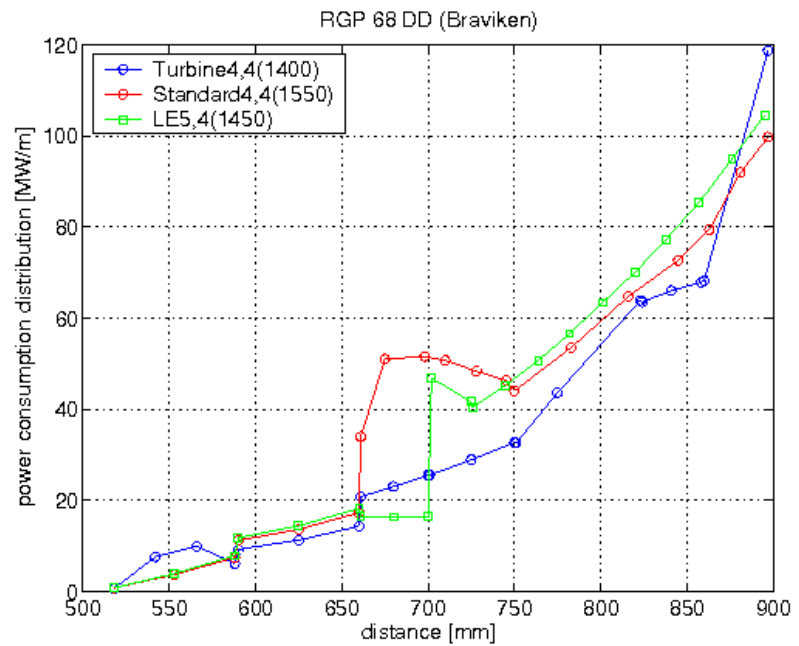

Fig 4. Power distribution along the segments radius. There is a large difference in how and where the power is distributed along the segment. Turbine segments have the smoothest power distribution and LE the most abrupt.

The power consumption distributions differ along the segments, Fig 4. The largest power consumption is at the segment periphery. Also, there is a large difference in power distribution around the distance of $700 \mathrm{~mm}$. This is where c- and p-segments are fitted together. In this particular area the smoothness of the power distribution might be an important factor for producing high quality pulp.

Another important aspect taken into account was the operating window for the different segment types. Calculations were made on all segment types in order to predict the intensity levels at different SEC and pressure inside the refiner. Results for the 


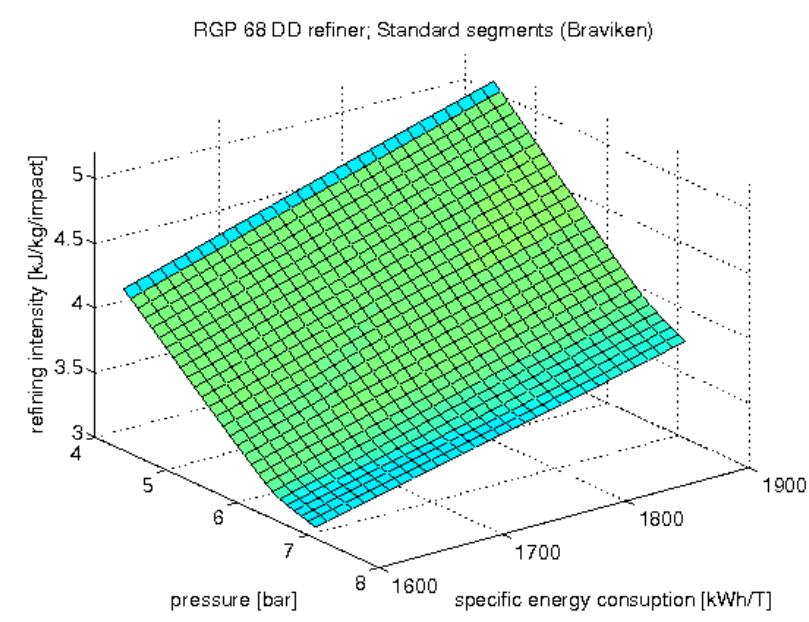

Fig 5. The intensity for the reference segment as a function of energy input and pressure.

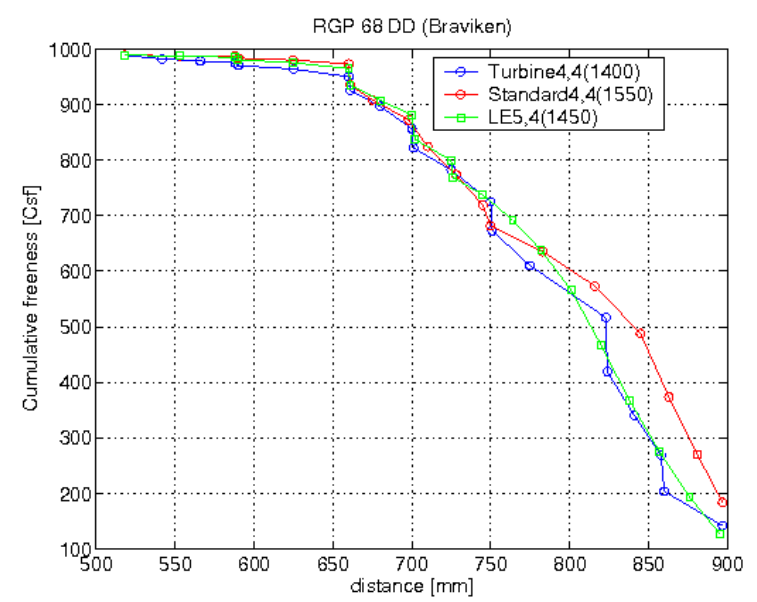

Fig 7. Cumulative CSF simulations for tested segment types.

reference and Turbine segment are shown in Fig 5 and Fig 6.

The effect of segment design on some pulp quality parameters can also be calculated. The CSF changes are simulated along the segment radius and CSF differences are shown between the different segment types, see Fig 7.

\section{Pulp quality}

Reference and Turbine segments had the same energy efficiency when comparing to same tensile index at SEC below $1600 \mathrm{kWh} / \mathrm{adt}$, Fig 8. The tensile index development is constant for Turbine segments above $1600 \mathrm{kWh} /$ adt, while it is increasing for the reference segment. The pulp produced with high intensity Turbine segment has shorter average fibre length and CSF than the reference segment, Figs 9 and 10. The CSF drop is around 50\% for the compared pulps.

The CSF drop was also simulated as shown in Fig 7. The reason for this fibre length and CSF drop is probably an effect of increased intensity, triggered by a feeding segment design that provides shorter

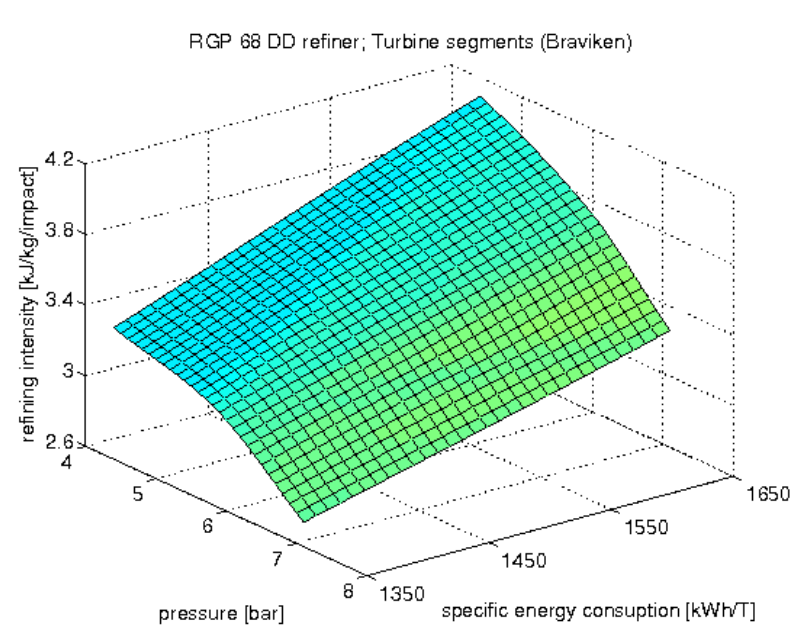

Fig 6. The intensity for the Turbine segment as a function of specific refining energy and pressure.

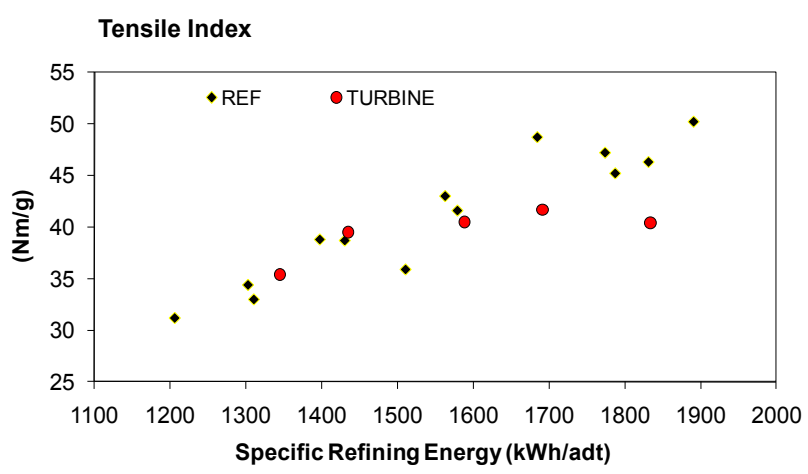

Fig 8. The tensile index development is similar for reference and Turbine segments. However, this tensile index development is constant for Turbine segments above 1600 kWh/adt.

minimum fibre residence time, Fig 2 and Fig 3. The CSF simulations agreed with the pulp result, Fig 7 and Fig 10.

A significant specific light scattering increase of $20 \%$ was observed when using Turbine segments, Fig 11.

\section{Segment design effect on the refiner}

It is interesting to compare the plate gap in the trials with Turbine and reference segments. Surprisingly the plate gap was larger when using the Turbine segment, Figs 12 and 13. This is in contradiction to earlier results (Muhic et al. 2010, Huhtanen et al. 2004, Huhtanen et al. 2009).

Another aspect that previously has shown to have a great potential regarding energy efficiency to reach a specific tensile index, when using standard segments, is high pressure inside the refiner. This was also tested in this study for Turbine segments; unfortunately we were unable to collect pulp samples from the blow line during the trial. The samples were collected from the stand pipe, and can therefore not directly be compared to the rest of the 
Average Fibre Length

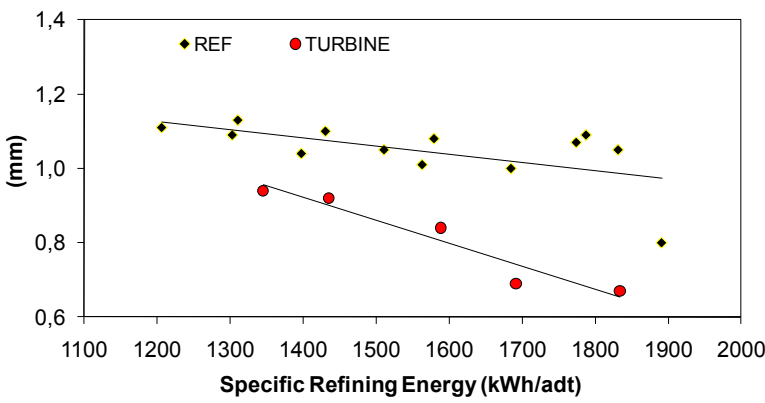

Fig 9. Turbine segments produce a pulp with shorter average fibre length compared to reference segments. The reduction is largest at high SEC.

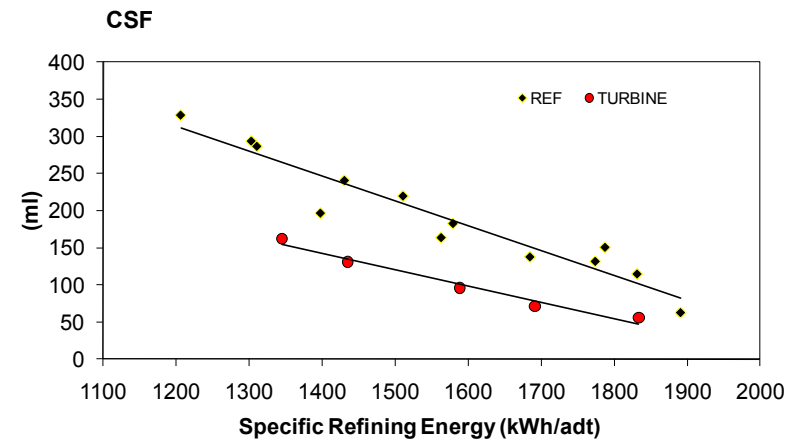

Fig 10. The CSF value is around $50 \%$ lower for Turbine segments compared to reference segments.

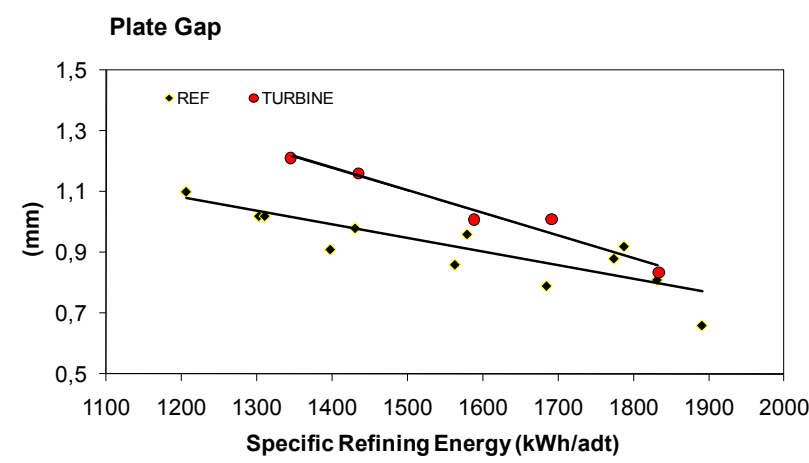

Fig 12. Plate gap was increased compared to similar tensile index when using Turbine segments.

blow line samples. However, refiner data shows a plate gap decrease of 5-8\% to reach a specific tensile index or SEC, when the refiner inlet and housing pressure was increased from 4.4 to 6.0 bar. The measured plate gap temperature increased by $\sim 5^{\circ} \mathrm{C}$ at same plate gap. Muhic et al. 2010 showed the same trends in trials with the reference segment. The result of that study was higher energy efficiency compared at same tensile index and light scattering. Even though the pulp results can not be scientifically presented, the refiner parameters indicate that high intensity segments (such as Turbine) and high pressure have good potential to increase energy

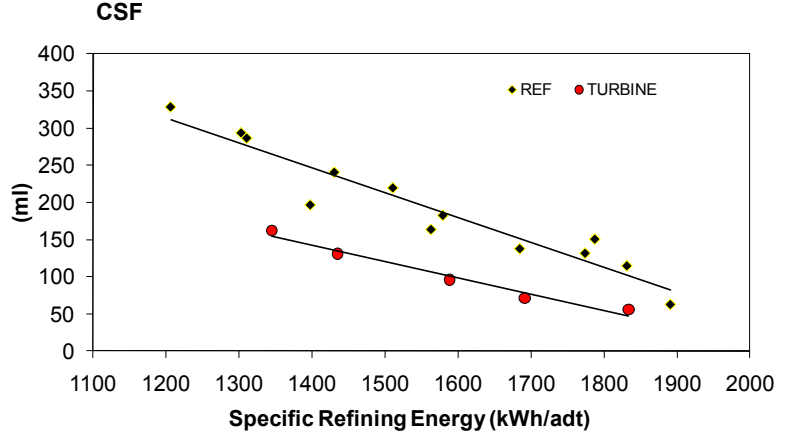

Fig 10. The CSF value is around $50 \%$ lower for Turbine segments compared to reference segments.

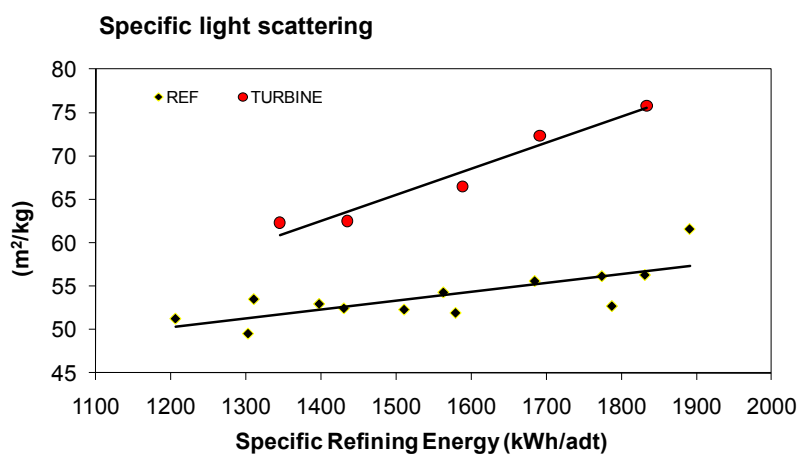

Fig 11. Specific light scattering increase with about $20 \%$ when using Turbine segments.

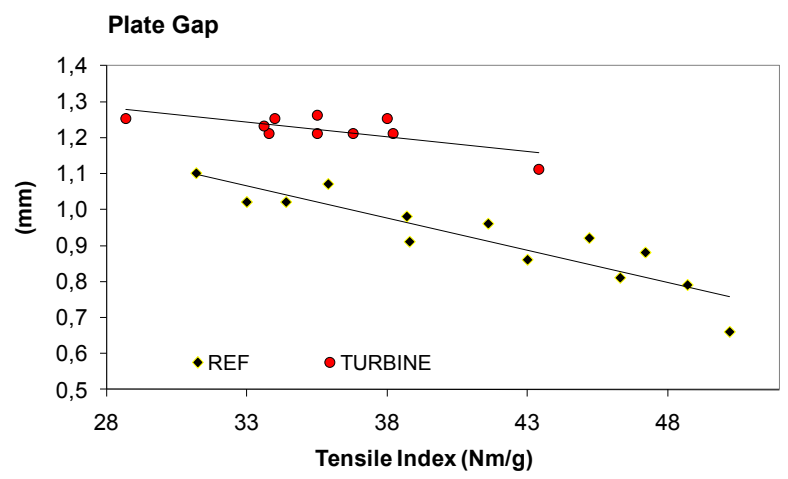

Fig 13. Compared to reference segments the plate gap increases at same tensile index when using Turbine segments.

efficiency at maintained strength properties and light scattering.

In Table $2 a-c$ measured temperature in the plate gap differs for the tested segments. This was also simulated before designing the segments, Fig 1 .

\section{Final discussion}

The trials made showed that segment design simulations and calculations can be a helpful tool in designing new segment patterns and predicting some pulp quality for a double disc refiner. For example, the abrupt power distribution change with LE segments (Fig 4) could have led to fibre cutting. In this study the tested feeding segments had 
perhaps too large bar angles to achieve the required pulp quality (tensile index $\sim 42$ with average fibre length above $0.9 \mathrm{~mm}$ ).

Beside the average fibre length decrease, the tear index (not shown) also drops when using Turbine segments. The question is how this fibre length and tear index drop affects the final pulp and paper quality and the runnability on the paper machine?

There were no noticeable differences in the final paper quality or the paper machine runnability during the Turbine segment trial period. This fact is perhaps a bit surprising. The first thought is that the pulp did not reach critical values in average fibre length, tear index, CSF etc. This could be true; however an average fibre length decrease of $20 \%$ and $50 \%$ CSF decrease should have a noticeable impact on the final paper quality. The second thought is that some other pulp properties increased or was maintained which positively affected (maintained) the paper quality. These questions will be further discussed in a later paper.

Another interesting thought is how the achieved high light scattering can be used. Usage of cheep chemicals (compared to electrical power) to gain strength properties could be combined with high light scattering in order to produce low-cost high quality pulp.

\section{Conclusions}

The use of Turbine segment (DN72N861-862) compared to a reference segments (DN72N816-817) resulted in;

$>$ Same tensile index at a certain SEC below $1600 \mathrm{kWh} / \mathrm{adt}$.

> Higher specific light scattering coefficient.

$>$ Limited operating window due to fibre cutting.

$>$ Lower SEC at same CSF.

$>$ Shorter average fibre length.

$>$ Larger plate gap.

$>$ Fluid dynamic models and segment pattern simulations can be used for predicting segment performance.

\section{Acknowledgements}

Our appreciation to all Braviken mill TMP operators, Jonas Franzén, Lennart Rohdén and Peter Bergqvist from Metso. Thanks to Veronica Bergenheim and Marie Nilzén for their laboratory help at Braviken. Thanks to Lars Hildén and Lorna Casson for their help with the manuscript.

Literature

Albert, K. P. (2001): Enhancing Refiner Performance and Lowering Energy Consumption with Overhung Plates, 6th PIRA International Conference, Toronto, Canada, 28-29 March, Proceedings
Atack, D., Stationwala, M., Karnis. (1984): What happens in refining, Pulp and Paper Canada, 85(12), pp119-124

Härkönen, E., Huusari, E., Ravila P. (2000): Residence time of fibre in a single disc refiner, Pulp and Paper Canada, T:330335

Härkönen, E., Kortelainen, J., Virtanen, J., Vuorio, P. (2003): Fiber development in TMP main line, International Mechanical Pulping Conference, Proceedings p. 171-178

Huhtanen, J-P. (2004): Modelling of Fibre Suspension Flows in Refiner and Other Papermaking Processes by Combining Non-Newtonian Fluid Dynamics and Turbulence, Tampere University of Technology, Publication 497, ISBN 952-15-1248-2

Huhtanen J.P., Karvinen R., Vikman K., Vuorio P. (2009): Determination of Freeness Reduction by Refining Intensity Definition Based on Fluid Dynamical Model, International Mechanical Pulping Conference, Proceedings p. 209-212

Huhtanen, J-P., Karvinen, R., Vikman, K., Vuorio, P. (2004): Theoretical Background of New Energy Saving Refiner Segments Design, PulPaper Conference, Energy and Carbon Management, Proceedings p. 111-118

Kure, K-A., Sabourin, M. J., Dahlqvist, G., Helle T. (1999): Adjusting Refining Intensity by Changing Refiner Plate Design and Rotational Speed- Effects on Structural Fibre Properties, Journal of Pulp and Paper Science, 26(10), J346-352

Miles, K.B., May, W.D. (1990): The flow of pulp in chip refiners, Journal of Pulp and Paper Science, Vol. 16(2) J63-72

Muhic, D., Sundström, L., Sandberg, C., Ullmar, M., Engstrand, P. (2010): Influence of Temperature on Energy Efficiency in Double Disc Chip Refining, NPPRJ vol.25 no.4/2010 p. 420-427

Pynnönen, T., Samboon, P. (2008): Performance of Abrasive Segments in Mechanical Pulp Refining. A Review, Papir ja Puu -Paper and Timber Vol 90/No.5

Sabourin, M., Xu E., Musselman, R. (1997): Evaluation of refiner disc speed, plate design and consistency on high pressure/low residence (RTS) TMP pulps, International Mechanical Pulping Conference, Proceedings p.241-249

Sanger, J.J., Ouellet, D. (2002): Factors Affecting the Shear Forces in High Consistency Refining, Journal of Pulp and Paper Science, 28(11):364-369

Stationwala, M.l., Atack, D., Karnis, A., (1992): Distribution and Motion of Pulp Fibres on Refiner Bar Surface, Journal of Pulp and Paper Science, Vol.18(4) J131-137

Vikman, K., Leskela, M., Pekkola, M., Vuorio, P., Tyrvainen, J., Huhtanen, J-P. (2003): New Refiner Segments Technology to Optimize the Aspen BCTMP Pulping Process, International Mechanical Pulping Conference, Proceedings p 91-95.

Vuorio, P., Bergqvist, P. (2003): New Refiner Segments Technology to Optimise Fibre Quality and Energy Consumption of Refiner Mechanical Pulp, TAPPSA J, pp 17-24 (C, P) (ISSN 1029-0109)

Manuscript received August 20, 2010 Accepted March 8, 2011 


\section{Appendix}

${ }^{*}$ All items protected by patent.

LowShive Segment TM (Reference segment)

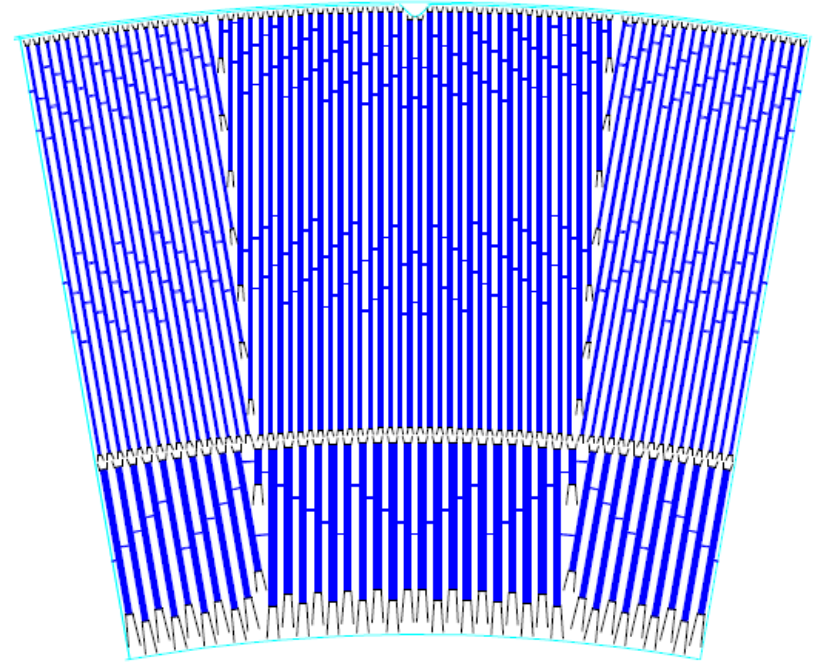

LE Segment/LowShive Segment TM

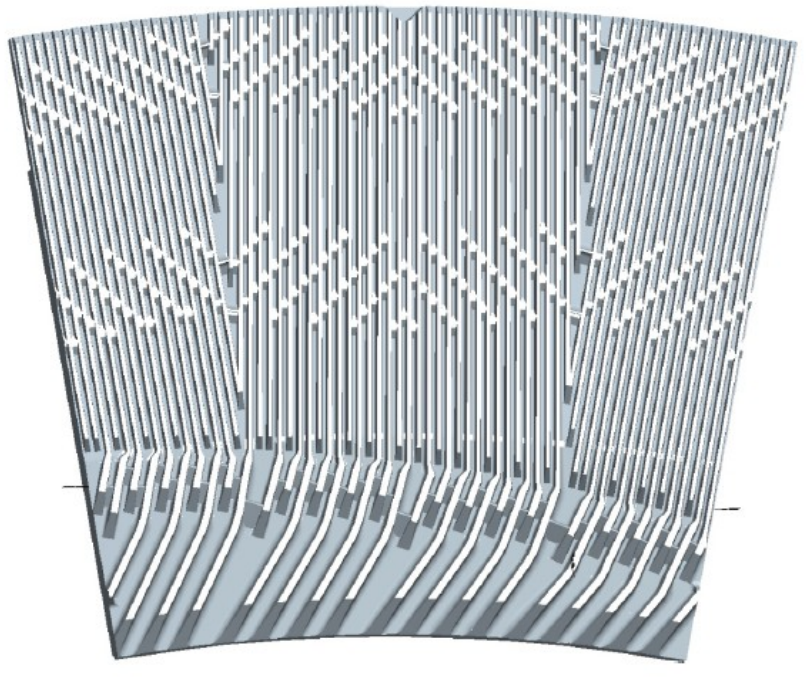

LE Segment/LowShive Segment/Turbine TM

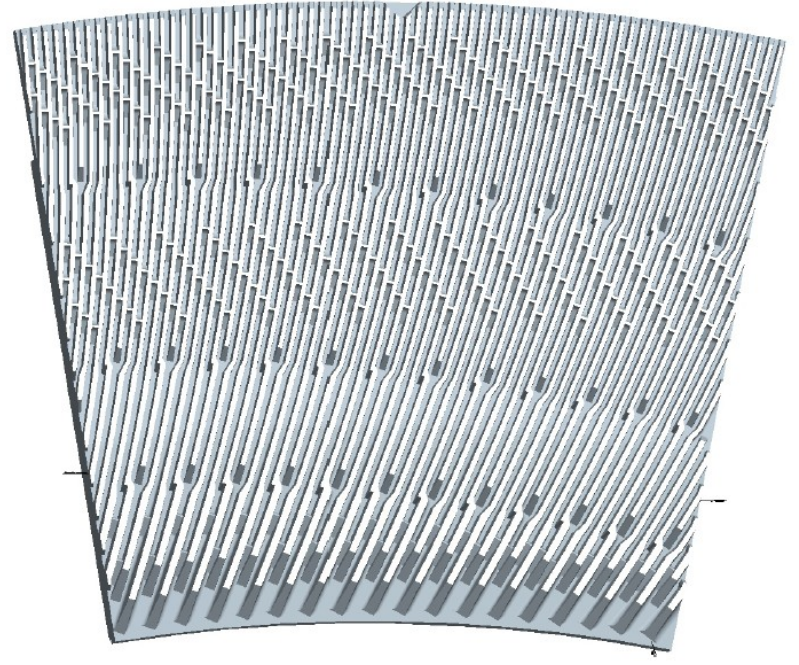

